MATHEMATICS OF COMPUTATION

Volume 73, Number 248, Pages 1849-1863

S 0025-5718(03)01614-4

Article electronically published on August 20, 2003

\title{
THE SYMMETRIC EIGENVALUE COMPLEMENTARITY PROBLEM
}

\author{
MARCELO QUEIROZ, JOAQUIM JÚDICE, AND CARLOS HUMES, JR.
}

\begin{abstract}
In this paper the Eigenvalue Complementarity Problem (EiCP) with real symmetric matrices is addressed. It is shown that the symmetric $(\mathrm{EiCP})$ is equivalent to finding an equilibrium solution of a differentiable optimization problem in a compact set. A necessary and sufficient condition for solvability is obtained which, when verified, gives a convenient starting point for any gradient-ascent local optimization method to converge to a solution of the (EiCP). It is further shown that similar results apply to the Symmetric Generalized Eigenvalue Complementarity Problem (GEiCP). Computational tests show that these reformulations improve the speed and robustness of the solution methods.
\end{abstract}

\section{INTRODUCTION}

The computation of eigenvalues is of crucial importance in a variety of practical problems in physics and engineering. Eigenvalues are related to the resonance frequency of structures and to stability of dynamical systems [4]. Real symmetric matrices appear in a large portion of these practical problems [13].

A classical approach to solving large-scale eigenvalue problems with real symmetric matrices is to apply unconstrained optimization techniques to the so-called Rayleigh quotient. This function has the property that every equilibrium point is an eigenvector with a corresponding eigenvalue given by the Rayleigh quotient.

The Eigenvalue Complementarity Problem (EiCP) appears in the study of static equilibrium states of finite dimensional mechanical systems with unilateral frictional contact [5] and takes the form

$$
(\operatorname{EiCP}) \text { : Find } \lambda>0, x \neq 0 \text { such that }\left\{\begin{array}{l}
w=(\lambda B-A) x, \\
w \geq 0 \\
x \geq 0 \\
w^{T} x=0 .
\end{array}\right.
$$

The reader is referred to [5, 14] for further details.

The purpose of this paper is to study the (EiCP) when $A$ and $B$ are symmetric matrices and $B$ is positive definite. This particular problem is shown to be equivalent to finding an equilibrium point of a generalized Rayleigh quotient satisfying $x \geq 0$ and $\lambda>0$, which can be solved using any gradient ascent method, from a convenient starting point. This result is akin to a classical result for Nonlinear

Received by the editor March 26, 2002 and, in revised form, January 23, 2003.

2000 Mathematics Subject Classification. Primary 90C33, 47A75; Secondary 90C30, 82B05.

The first author was supported by FAPESP Grant Nos. 97/06227-2 and 02/01351-7.

The second author was supported by FCT project POCTI/35059/MAT/2000. 
Complementarity Problems (NCP) with symmetric jacobians, although the classical result is not applicable to an NCP reformulation of the (EiCP) (Section 4). The result is extended to a generalization of the symmetric (EiCP) of practical interest, the symmetric (GEiCP). Computational tests show that these reformulations improve the speed and robustness of the solution methods. Some preliminary research on the asymmetric case is also presented.

The structure of the paper is as follows. In Section 2 the classical eigenvalue problem is discussed, with emphasis on symmetric matrices. The relationship of this problem with the optimization of the Rayleigh quotient is established, showing that the eigenpairs can be computed with gradient related methods.

In Section 3 the Eigenvalue Complementarity Problem (EiCP) and the Generalized Eigenvalue Problem (GEiCP) are introduced. Some general results concerning the solvability of these problems and the number of complementary eigenvalues are presented. It is shown that in general these problems are NP-hard. In this paper the term solvability when applied to the (EiCP) or ( $\mathrm{GEiCP})$ means the existence of a complementary solution.

The symmetric case is studied in Section 4 It is shown that in this case both the (EiCP) and the (GEiCP) are equivalent to finding equilibrium points of optimization problems and therefore are relatively easy to solve. Computational experience is reported in Section 5.

Some preliminary work on the asymmetric case is presented in Section 6, and the last section (Section 7) presents some conclusions and future work.

\section{EigenVALUe PROBlems AND OPtimization}

Given a matrix $A \in \mathbb{R}^{n \times n}$, the classical Eigenvalue Problem (EiP) is

$$
\text { (EiP): Find } \lambda \in \mathbb{R}, x \neq 0 \text { such that } A x=\lambda x \text {. }
$$

A necessary and sufficient condition for the existence of a solution with $x \neq 0$ is that the kernel of $(A-\lambda I)$ be nontrivial, and this occurs if and only if $\lambda$ is a root of the polynomial equation $\operatorname{det}(A-\lambda I)=0$. Such values of $\lambda$ are referred to as the solutions of the (EiP) and correspond to the eigenvalues of $A$. Since the polynomial above has degree $n$, the (EiP) has at most $n$ distinct solutions.

If $\bar{\lambda}$ is an eigenvalue of $A$, computing a corresponding eigenvector amounts to finding a nontrivial solution of the linear system $(A-\bar{\lambda} I) x=0$. Since this system is homogeneous, $x$ is an eigenvector corresponding to $\bar{\lambda}$ if and only if $\alpha x$ is also an eigenvector corresponding to $\bar{\lambda}$, for any $\alpha \neq 0$. An extensive treatment of properties and algorithms for the (EiP) can be found in [13] and [4].

A classical approach to solving large-scale (EiP) problems with real symmetric matrices is to apply unconstrained optimization techniques to the Rayleigh quotient

$$
\lambda(x)=\frac{x^{T} A x}{x^{T} x} .
$$

This quotient is only defined for $x \neq 0$. The gradient of the Rayleigh quotient is

$$
\nabla \lambda(x)=\frac{2}{x^{T} x}[A-\lambda(x) I] x .
$$

Since $\nabla \lambda(x)=0$ if and only if $[A-\lambda(x) I] x=0$, any equilibrium point $(x, \lambda(x))$ of the Rayleigh quotient is a solution of the (EiP). The expression of the gradient of the Rayleigh quotient above is only valid when $A$ is symmetric. If $A \neq A^{T}$, the 
correct expression is $\nabla \lambda(x)=\frac{1}{x^{T} x}\left[A+A^{T}-2 \lambda(x) I\right] x$, and the relationship between equilibrium points and solutions of the (EiP) ceases to hold.

The Generalized Eigenvalue Problem (GEiP) is often referred to in the literature: given $A, B \in \mathbb{R}^{n \times n}$,

(GEiP): Find $\lambda \in \mathbb{R}, x \neq 0$ such that $A x=\lambda B x$.

The $\lambda$-solutions of this problem are referred to as general eigenvalues of $(A, B)$. This problem is in general more difficult than the (EiP) [13. When $B$ is symmetric positive definite and $A$ is symmetric, a generalized Rayleigh quotient can be defined as

$$
\lambda(x)=\frac{x^{T} A x}{x^{T} B x}
$$

and the (GEiP) can also be solved by optimization. The gradient in this case is

$$
\nabla \lambda(x)=\frac{2}{x^{T} B x}[A-\lambda(x) B] x,
$$

and the equilibrium points of $\lambda(x)$ correspond to general eigenvectors and eigenvalues of $(A, B)$.

The lemma below presents two fundamental properties of the generalized Rayleigh quotient. Its proof is straightforward.

Lemma 1. For all $x \neq 0$ the following equalities hold:

(1) $\lambda(\alpha x)=\lambda(x), \forall \alpha>0$;

(2) $x^{T} \nabla \lambda(x)=0$.

Since the case $B=I$ and $B$ symmetric positive definite are subject to the same approach with respect to the optimization of the Rayleigh quotient, all the complementary problems introduced in the next section are formulated with two matrices $(A, B)$, where $B$ is required to be positive definite.

Other functions whose equilibrium points correspond to pairs of eigenvalues and eigenvectors exist for special classes of matrices. For instance, the functions $S_{A}(x)=x^{T} x-2 \sqrt{x^{T} A x}, P_{A}(x)=\left(x^{T} x\right)^{2}-2 x^{T} A x$ and $L_{A}(x)=x^{T} x-\ln \left(x^{T} A x\right)$, for a symmetric positive definite matrix $A$, have their equilibrium points corresponding to eigenvectors of $A$, whereas $S_{A}(\cdot)$ and $P_{A}(\cdot)$ also have the origin as an equilibrium point. The papers [9, 1, 2] present theoretical results and computational experience comparing these functions to the Rayleigh quotient for symmetric positive definite matrices. The present work does not address these functions in order to keep the framework more general.

\section{The eigenValue COMPLEMENTARity PRoblem}

As stated in the Introduction, the Eigenvalue Complementarity Problem (EiCP) appeared in the study of static equilibrium states of mechanical systems with unilateral friction, in [5]. It may be expressed as

$$
(\mathrm{EiCP}) \text { : Find } \lambda>0, x \neq 0 \text { such that }\left\{\begin{array}{l}
w=(\lambda B-A) x \\
w \geq 0 \\
x \geq 0 \\
w^{T} x=0
\end{array}\right.
$$

where $B$ is a positive definite matrix. Note that any solution with $w=0$ corresponds to a solution of the (GEiP). 
The Generalized Eigenvalue Complementarity Problem $(\mathrm{GEiCP})_{J}$ is

$$
(\mathrm{GEiCP})_{J} \text { : Find } \lambda>0, x \neq 0 \text { such that }\left\{\begin{array}{l}
w=(\lambda B-A) x \\
w_{\bar{J}}=0, \\
w_{J} \geq 0 \\
x_{J} \geq 0 \\
w_{J}^{T} x_{J}=0,
\end{array}\right.
$$

where $B$ is a positive definite matrix, $J \subseteq\{1,2, \ldots, n\}$ is given, and $\bar{J}=$ $\{1,2, \ldots, n\} \backslash J$. The (EiCP) is clearly the particular case of the $(\mathrm{GEiCP})_{J}$ with $J=\{1,2, \ldots, n\}$.

For any solution $(\lambda, x, w)$, the value $\lambda$ is called a (general) complementary eigenvalue of $(A, B)$, and $x$ is a corresponding (general) complementary eigenvector. Since the set of complementary eigenvectors of a given complementary eigenvalue is a cone, there is no loss of generality in restricting the problem to finding solutions satisfying $\|x\|_{2}=1$, which replaces the constraint $x \neq 0$. In the case of the (EiCP) the linear constraint $\|x\|_{1}=e^{T} x=1$ can be considered instead of $\|x\|_{2}=1$, since $x \geq 0$.

It is easy to see that any solution of the (EiCP) (or (GEiCP)) with $w=0$ is a positive eigenvalue of $(A, B)$ with a corresponding eigenvector satisfying some sign constraints. The following two results, originally by Seeger [14, have been slightly expanded to include the (GEiCP) case.

Proposition 2 (Seeger). For any solution $(\lambda, x)$ of $(G E i C P)_{J}$, there is a set $I$ satisfying $\bar{J} \subseteq I \subseteq\{1,2, \ldots, n\}$, such that $\lambda$ is a positive eigenvalue of $\left(A_{I I}, B_{I I}\right)$ and $x_{I}$ is a corresponding eigenvector satisfying $x_{J \cap I} \geq 0$.

Proof. Set $I=\left\{i \mid w_{i}=0\right\}$. Then $I \supseteq \bar{J}$, and $x_{\bar{I}}=0$, by complementarity. Therefore $A_{I I} x_{I}=\lambda B_{I I} x_{I}$.

For the $(\mathrm{EiCP})$, this result says that given a solution $(\lambda, x), \lambda$ is a positive eigenvalue of $\left(A_{I I}, B_{I I}\right)$ and $x_{I}$ is a corresponding nonnegative eigenvector. A direct corollary says that the number of $\lambda$-solutions of the (EiCP) and (GEiCP) is finite.

Proposition 3 (Seeger). The (EiCP) has at most $n 2^{n-1}$ distinct $\lambda$-solutions. The $(G E i C P)_{J}$ has at most $(2 n-|J|) 2^{|J|-1}$ distinct $\lambda$-solutions.

Proof. Consider first the $(\mathrm{GEiCP})_{J}$. There are $2^{|J|}$ possible subsets $I$ such that $\bar{J} \subset I \subset\{1,2, \ldots, n\}$. For each possible subset $I$ there are at most $|I|$ distinct $\lambda$-solutions, namely the eigenvalues of $B_{I I}^{-1} A_{I I}$. Therefore the total number of $\lambda$-solutions is at most

$$
\begin{aligned}
\sum_{i=0}^{|J|}(|\bar{J}|+i)\left(\begin{array}{c}
|J| \\
i
\end{array}\right) & =(n-|J|) \sum_{i=0}^{|J|}\left(\begin{array}{c}
|J| \\
i
\end{array}\right)+\sum_{i=1}^{|J|} \sum_{k=i}^{|J|}\left(\begin{array}{c}
|J| \\
k
\end{array}\right) \\
& =(n-|J|) 2^{|J|}+|J| 2^{|J|-1} \\
& =(2 n-|J|) 2^{|J|-1} .
\end{aligned}
$$

Since the (EiCP) is the particular instance of the $(\mathrm{GEiCP})_{J}$ with $J=\{1,2, \ldots, n\}$, the number of $\lambda$-solutions of the (EiCP) is at most

$$
(2 n-|J|) 2^{|J|-1}=n 2^{n-1} .
$$

The complete set of solutions of both (EiCP) and (GEiCP) can be obtained via complete enumeration, as follows. 
Complete set of solutions of $(\mathrm{GEiCP})_{\mathrm{J}}$.

(1) For each subset $I$ such that $\bar{J} \subseteq I \subseteq\{1,2, \ldots, n\}$ compute the set $\Lambda(I)$ of eigenvalues of $B_{I I}^{-1} A_{I I}$.

(2) For each $\bar{\lambda} \in \Lambda(I)$ such that $\bar{\lambda}>0$, try to compute $x_{I}$ such that

$$
\left\{\begin{array}{l}
A_{I I} x_{I}=\bar{\lambda} B_{I I} x_{I}, \\
A_{J I} x_{I} \geq \bar{\lambda} B_{J I} x_{I}, \\
x_{I \cap J} \geq 0 \\
\left\|x_{I}\right\|_{2}=1 .
\end{array}\right.
$$

If such a solution exists, then $x=\left(x_{I}, 0\right)$ is a general complementary eigenvector corresponding to the general complementary eigenvalue $\bar{\lambda}$.

The soundness of this method is a direct consequence of Proposition 2 This algorithm also provides a necessary and sufficient test for solvability of a given (GEiCP). The following proposition shows that any necessary and sufficient test for the solvability of a general (GEiCP) is doomed to be computationally expensive.

Proposition 4. The solvability of the $(G E i C P)_{J}$ is an NP-complete decision problem.

Proof. Any solution $(\lambda, x)$ of the $(\mathrm{GEiCP})_{J}$ satisfies $x_{J} \geq 0$ and

$$
x^{T} A x=x^{T}(\lambda B x-w)=\lambda x^{T} B x-x^{T} w=\lambda x^{T} B x>0,
$$

since $x \neq 0, \lambda>0$ and $B$ is positive definite. Therefore, deciding if the (GEiCP $)_{J}$ is solvable is at least as difficult as deciding if there exists an $x$ such that $x_{J} \geq 0$ and $x^{T} A x>0$, for a given matrix $A$. The latter problem is NP-complete [11, Theorem $2.20]$. Since the solvability of the $(\mathrm{GEiCP})_{J}$ is in NP (i.e., one can polynomially check whether a given pair $(\lambda, x)$ is a solution of the $\left.(\mathrm{GEiCP})_{J}\right)$, the result of the proposition follows.

It follows that solving the (GEiCP) is in general an NP-hard problem. Despite this fact, for some classes of matrices the solvability of the corresponding (EiCP) or ( $\mathrm{GEiCP})$ can be answered easily.

Lemma 5. If $A$ is negative semi-definite (i.e., $x^{T} A x \leq 0$ for all $x$ ), the corresponding $(\mathrm{GEiCP})$ is unsolvable.

Proof. Since $A$ is negative semi-definite, it follows that $A_{I I}$ is negative semi-definite for all $I \subseteq\{1,2, \ldots, n\}$. Therefore $x_{I}^{T} A_{I I} x_{I} \leq 0$, for any $x$, and the problem is unsolvable.

Lemma 6. If $A$ is positive $\left(A_{i j}>0, \forall i, j\right)$ and $B=I$, then the (EiCP) has a solution $\bar{\lambda}>0$, with a corresponding eigenvector $\bar{x}>0$. Moreover, if $A$ is symmetric, then the solution $\bar{\lambda}$ is unique.

Proof. Since $A$ is positive, any feasible solution $\bar{x} \neq 0$ has to satisfy $\bar{x}>0$, for otherwise $\bar{x}_{i}=0$ and $\bar{x} \neq 0$ implies $\bar{w}_{i}=\bar{\lambda} \bar{x}_{i}-A_{i} \bar{x}=-A_{i} \bar{x}<0$. By complementarity, $\bar{w}=0$, and therefore this (EiCP) is equivalent to the (EiP). The existence of a solution of the (EiP) with a positive matrix is Perron's Theorem [12, Theorem 6.1.2]. The uniqueness in the symmetric case is verified as follows. Let $\hat{\lambda} \neq \bar{\lambda}$, and consider a vector $\hat{x}$ such that $A \hat{x}=\hat{\lambda} \hat{x}$. Then,

$$
(\hat{\lambda}-\bar{\lambda}) \hat{x}^{T} \bar{x}=(A \hat{x})^{T} \bar{x}-\hat{x}^{T}(A \bar{x})=\hat{x}^{T} A^{T} \bar{x}-\hat{x}^{T}(A \bar{x})=0,
$$


and since $\hat{\lambda} \neq \bar{\lambda}$, it follows that $\hat{x}^{T} \bar{x}=0$. Since $\bar{x}>0$, there is an index $i$ such that $\hat{x}_{i}<0$, and therefore $\hat{x}$ is not a solution of the (EiP).

Lemma 7. If there is some index $j$ such that $A_{j j}>0$ and $A_{i j} \leq 0, \forall i \neq j$, then $(\lambda, x)$ is a solution of the (EiCP), with $\lambda=A_{j j}$ and $x=e^{j}$.

Proof. Trivial.

In particular this property holds for the well-known class of nonsingular Mmatrices, which are defined as P-matrices (all principal minors are positive) with nonpositive off-diagonal elements.

The following section is devoted to the special case of the Eigenvalue Complementarity Problems with symmetric matrices. Some additional properties of the general (GEiCP) are presented in Section 6.

\section{The symmetric Eigenvalue Complementarity Problem}

In this section the symmetric versions of (EiCP) and (GEiCP) are studied. Some properties of these problems are derived, including a necessary and sufficient condition for solvability. When this condition is verified, the problem can be solved by a variety of well-known methods in differentiable nonlinear programming.

For clarity of exposition, the results are presented separately for the (EiCP) and the (GEiCP).

The symmetric (EiCP). An interesting known property of the symmetric (EiCP) regards the number of $\lambda$-solutions; in this case a smaller upper bound than the one presented in the general case can be found. The proof of this result can be found in [14.

Proposition 8 (Seeger). The number of $\lambda$-solutions of the symmetric (EiCP) is at most $2^{n}-1$.

The symmetric (EiCP) is closely related to the classical eigenvalue problem. Since $x \neq 0$ for any solution, the complementarity condition $w^{T} x=0$ may be rewritten as $x^{T}(\lambda B x-A x)=0$, or equivalently,

$$
\lambda(x)=\frac{x^{T} A x}{x^{T} B x} .
$$

This is again the generalized Rayleigh quotient. Analogously to the classical case, equilibrium points of the Rayleigh quotient in the nonnegative orthant with $\lambda(x)>$ 0 are solutions of the (EiCP). This is the main result concerning the practical solution of the symmetric (EiCP).

Proposition 9. The symmetric (EiCP) is equivalent to

$$
(O E i C P) \begin{cases}\max & \lambda(x) \\ \text { s.t. } & x \geq 0, \\ & e^{T} x=1,\end{cases}
$$

in the sense that every equilibrium solution $x$ of (OEiCP) with $\lambda(x)>0$ is a solution of the (EiCP). 
Proof. The optimization problem (OEiCP) is equivalent to

$$
\max _{x \in \mathbb{R}^{n}} \inf _{\substack{u \geq 0 \\ \alpha \in \mathbb{R}}} \frac{x^{T} A x}{x^{T} B x}+u^{T} x+\alpha\left(e^{T} x-1\right) .
$$

Any equilibrium solution of this problem satisfies the Kuhn-Tucker conditions

$$
\left\{\begin{array}{l}
u+\alpha e=\frac{2}{x^{T} B x}[\lambda(x) B-A] x, \\
u \geq 0 \\
x \geq 0 \\
u^{T} x=0 \\
e^{T} x=1
\end{array}\right.
$$

By performing the scalar product of the first equation with $x$ and noticing that $u^{T} x=0, e^{T} x=1$, and $\lambda(x)=\frac{x^{T} A x}{x^{T} B x}$, it follows that $\alpha=0$. The resulting system of equations corresponds closely to the (EiCP), with $w=\frac{x^{T} B x}{2} u$. Since $B$ is positive definite and $\lambda(x)>0$, it follows that $(\lambda, x, w)$ is a solution of the $(\mathrm{EiCP})$, and the result follows.

The conclusion that $\alpha=0$ in the proof of Proposition 9, though apparently nonintuitive, may be motivated by the homogeneity of $\lambda(\cdot)$ and the orthogonality between $x$ and $\nabla \lambda(x)$ (Lemma 1). Consider, for instance, an equilibrium point of the (OEiCP) satisfying $x>0$. By the homogeneity of $\lambda(\cdot)$, the projection of $\nabla \lambda(x)$ on the half-line $\{\alpha x \mid \alpha \geq 0\}$ vanishes. By the equilibrium property with respect to the constraint $e^{T} x=1$, the projection of $\nabla \lambda(x)$ on the affine manifold $\left\{x \mid e^{T} x=1\right\}$ also vanishes. Therefore, $\nabla \lambda(x)=0$, and so the Lagrange multiplier $\alpha$ in the Kuhn-Tucker conditions becomes superfluous.

Proposition 9 resembles a classical result for nonlinear complementarity problems of the form

$$
\left\{\begin{array}{l}
x \geq 0 \\
F(x) \geq 0 \\
x^{T} F(x)=0,
\end{array}\right.
$$

with $\nabla F(x)$ symmetric for all $x$. In fact, such problems can be solved as optimization problems because $F(x)$ is shown to correspond to the gradient of a primitive function $f(x)$, whose equilibrium points are solutions of the NCP [7]. In the present case the constraint $e^{T} x=1$ ( or $x \neq 0$ ) breaks the symmetry of the enlarged NCP, obtained by considering an additional complementary variable $\alpha$ satisfying

$$
\left\{\begin{array}{l}
w=(B-\mu A) x, \\
\alpha=-1+e^{T} x \\
x, w \geq 0 \\
\mu, \alpha \geq 0 \\
w^{T} x=\alpha \mu=0
\end{array}\right.
$$

where $\mu=\frac{1}{\lambda}$ with respect to the original problem. In this setting, the hypothesis of the symmetric jacobians does not hold. Therefore, it is not possible to use the classical theorem to conclude that the NCP above is solvable as an optimization problem.

From the reformulation of the (EiCP) as a nonlinear programming problem several conclusions are drawn. The first one is a characterization of solvability of the $(\mathrm{EiCP})$. 
Proposition 10. (EiCP) is solvable if and only if there exists some $x \geq 0$ such that $x^{T} A x>0$.

Proof. First suppose that the (EiCP) is solvable. If $(\lambda, x)$ is any solution of the (EiCP), then $x \geq 0$ and $x^{T} A x=\lambda x^{T} B x>0$, since $x \neq 0$ and $B$ is positive definite.

Suppose, on the other hand, that there is a $\hat{x} \geq 0$ such that $\hat{x}^{T} A \hat{x}>0$. Since $\lambda(x)$ is a continuous function (for $x \neq 0$ ) and the set $X=\left\{x \geq 0 \mid e^{T} x=1\right\}$ is compact, there exists a $\bar{x} \in X$ such that $\lambda(\bar{x}) \geq \lambda(x), \forall x \in X$. This is an equilibrium point for the (OEiCP), and in particular, $\lambda(\bar{x}) \geq \lambda(\hat{x})>0$. Therefore, $\bar{x}$ is a solution of the (EiCP).

For the practical solution of the symmetric (EiCP), any ascent gradient method, with an initial solution $\hat{x} \in X$ such that $\hat{x}^{T} A \hat{x}>0$ obtains an equilibrium point which is a solution of the problem. The nonconvexity of the objective function is actually not a problem, since the global optimization problem (OEiCP) need not be solved. However, finding such an initial point is an NP-complete problem (Proposition 4). But for a very large class of matrices this problem is trivial, as the next proposition shows. For these matrices, an initial point can be found by inspection, and a solution of the (EiCP) is thereafter easily obtained.

Proposition 11. Suppose that the matrix A satisfies one of the conditions

(1) $\exists i: A_{i i}>0$;

(2) $\exists i, j: A_{i i}=0, A_{j j} \leq 0$ and $A_{i j}>0$;

(3) $A \geq 0, A \neq 0$;

(4) $A$ is an $S$-matrix $(\exists x \geq 0: A x>0)$.

Then a point $\hat{x} \in X$ such that $\hat{x}^{T} A \hat{x}>0$ can be easily obtained and the corresponding (EiCP) is solvable.

Proof. (1) Let $\hat{x}=e^{i}$; then $\hat{x}^{T} A \hat{x}=A_{i i}=1$.

(2) Let $\hat{x}_{j}=1, \hat{x}_{i}=\frac{1-A_{j j}}{2 A_{i j}}$, and $\hat{x}_{k}=0, \forall k \neq i, j$; then $\hat{x}^{T} A \hat{x}=A_{j j} \hat{x}_{j}^{2}+$ $2 A_{i j} \hat{x}_{i} \hat{x}_{j}=1$.

(3) If $A \geq 0, A \neq 0$, then $\hat{x}=e$ is such that $\hat{x}^{T} A \hat{x}>0$.

(4) If $A$ is an $S$-matrix, then there exists a $\hat{x} \geq 0$ such that $A \hat{x}>0$. It follows that $\hat{x}^{T} A \hat{x}>0$. Such a $\hat{x}$ can be found by solving the following linear program:

$$
(\mathrm{LP}) \begin{cases}\max & y \\ \text { s.t. } & A x-y e \geq 0 \\ & e^{T} x=1 \\ & x \geq 0 \\ & y \in \mathbb{R} .\end{cases}
$$

This problem is always feasible, and since the variable $x$ lies in a compact set and $y \leq \min \left\{(A x)_{i}\right\}$, an optimal solution exists. Now since $A$ is an $S$-matrix, the optimal solution $(\hat{x}, \hat{y})$ satisfies $\hat{y}>0, \hat{x} \geq 0$ and so $\hat{x}^{T} A \hat{x} \geq$ $\hat{y} \hat{x}^{T} e=\hat{y}>0$.

Note that condition (1) of the previous result ( $A$ has at least one positive diagonal entry) defines a very large class of matrices that includes nontrivial positive semidefinite matrices (i.e., $x^{T} A x \geq 0$ for all $x$ ) and strictly copositive matrices (i.e., $x^{T} A x>0$ for all $0 \neq x \geq 0$ ). Condition (2) includes nontrivial copositive matrices (i.e., $x^{T} A x \geq 0$ for all $x \geq 0$ ). 
For the design of an initialization algorithm, besides applying the tests indicated above, additional heuristics may be implemented. One of these is to find local maxima of the nonconvex quadratic problem

$$
(\mathrm{QP}) \begin{cases}\max & x^{T} A x \\ \text { s.t. } & e^{T} x=1, \\ & x \geq 0\end{cases}
$$

from a few random starting points. Other tests are given by Lemmas [5, 6] and 7 in the previous section. A general algorithmic approach is to solve a GLCP (Generalized LCP; see [8]) as below.

Proposition 12. Given a matrix $A$, there exists a vector $x \geq 0$ such that $e^{T} x=1$ and $x^{T} A x \geq \varepsilon>0$ if and only if the system

$$
\left\{\begin{array}{l}
u+\alpha e+A x=0 \\
-\alpha \geq \varepsilon \\
u \geq 0 \\
x \geq 0 \\
u^{T} x=0 \\
e^{T} x=1
\end{array}\right.
$$

is solvable.

Proof. This system corresponds to the Kuhn-Tucker conditions of the (QP) and the additional constraint $-\alpha \geq \varepsilon$. This additional constraint is equivalent to $x^{T} A x \geq \varepsilon$, which can be seen by taking the inner product of the first equation with $x$ and noticing that $u^{T} x=0$ and $e^{T} x=1$. Since the feasible set of the (QP) is compact, the existence of an $x \geq 0$ such that $e^{T} x=1$ and $x^{T} A x \geq \varepsilon>0$ is equivalent to the existence of an equilibrium point of the (QP) satisfying the same conditions, which is equivalent to the GLCP above.

Computational methods for this problem, such as the SLCP method, can be found in [8].

The symmetric (GEiCP). The main results from this section can be rephrased in the context of the symmetric (GEiCP). Once again the complementarity constraint $x^{T} w=0$ allows $\lambda$ to be expressed as a function of $x$,

$$
\lambda(x)=\frac{x^{T} A x}{x^{T} B x} .
$$

Since $x$ is not necessarily nonnegative (only $x_{J}$ is nonnegative), the condition $x \neq 0$ cannot be replaced by $e^{T} x=1$. The cumbersome constraint $\|x\|_{2}=1$ is adopted for the sake of theory only. It is later dropped from the computational model, which is able to handle this constraint implicitly. The symbol $\|\cdot\|$ without sub-index is used to denote the euclidean norm.

Proposition 13. The symmetric (GEiCP) is equivalent to

$$
(O G E i C P) \begin{cases}\max & \lambda(x) \\ \text { s.t. } & x_{J} \geq 0, \\ & \|x\|=1,\end{cases}
$$

in the sense that every equilibrium solution $x$ of $(O G E i C P)$ with $\lambda(x)>0$ is a solution to the (GEiCP). 
Proof. The proof is analogous to that of Proposition 9.

The difficulty of the optimization problem (OGEiCP) is revealed by the nonconcavity of the objective function (maximization problem) and by the nonconvex constraint $\|x\|=1$. The nonconvexity of the objective function is not really a problem, since one looks after equilibrium solutions, and not globally optimal solutions, exactly as in the (EiCP) case. The feasible region of (OGEiCP) is a spherical surface with sign constraints, and therefore projection methods can be easily implemented via renormalization of iterates. See, for instance, the projected steepest ascent method below and Proposition 15 .

The solvability condition for the (GEiCP) is analogous to the one for the (EiCP).

Proposition 14. $(G E i C P)_{J}$ is solvable if and only if there exists some $x \in \mathbb{R}^{n}$ such that $x_{J} \geq 0$ and $x^{T} A x>0$.

It is clear that if the $(\mathrm{EiCP})$ is solvable, then the $(\mathrm{GEiCP})_{J}$ is solvable. But the solvability of the $(\mathrm{GEiCP})_{J}$ holds for a larger class of matrices. For instance, the $(\mathrm{GEiCP})_{J}$ with $n=2, J=\{1\}, B=I$ and

$$
A=\left[\begin{array}{cc}
0 & -\frac{1}{2} \\
-\frac{1}{2} & -1
\end{array}\right]
$$

is solvable, since $x=(2,-1)^{T}$ is such that $x_{J}=2 \geq 0$ and $x^{T} A x=1>0$. A solution is obtained with $x=(0.8844,-0.3663)$ and $\lambda=0.2071$. However, the corresponding (EiCP) is unsolvable, since $-A$ is copositive.

For the practical solution of the symmetric $(\mathrm{GEiCP})_{J}$, a starting point satisfying $x_{J} \geq 0$ and $x^{T} A x>0$ is needed, as well as an ascent gradient method for the optimization problem (OGEiCP).

The initialization for the solution of the $(\mathrm{GEiCP})_{J}$ is akin to the $(\mathrm{EiCP})$ case. The heuristics of Lemmas [5, 6] and 7] and Proposition [1] are applicable in this framework as well, and a general method for the initialization is to solve the GLCP below [8].

$$
\left\{\begin{array}{l}
u+\alpha e+A x=0 \\
-\alpha \geq \varepsilon \\
u_{J} \geq 0 \\
x_{J} \geq 0 \\
u_{J}^{T} x_{J}=0 \\
u_{J}=0 \\
e_{J}^{T} x_{J}=1 .
\end{array}\right.
$$

In this problem $x \neq 0$ is replaced by $e_{J}^{T} x_{J}=1$. This is not done in the optimization problem (OGEiCP) to ensure compactness of the feasible region, which is needed in the convergence proof of the method presented below.

Given a good starting point, a gradient-ascent method can be easily adapted to handle the constraint $\|x\|=1$ implicitly, by renormalizing every iterate of the sequence. As an example, the canonic steepest ascent method is slightly rewritten to solve the symmetric (GEiCP), using Armijo's step-length choice.

Projected Steepest Ascent Method for the symmetric $(G E i C P)_{J}$.

(0) Let $\varepsilon \in(0,1)$

Let $x^{0}$ satisfy $x_{J}^{0} \geq 0,\left\|x^{0}\right\|=1$ and $\left(x^{0}\right)^{T} A x^{0}>0$.

Let $k \longleftarrow 0$. 
(1) Compute $\nabla \lambda\left(x^{k}\right)=\frac{2}{\left(x^{k}\right)^{T} B x^{k}}\left[A-\lambda\left(x^{k}\right) B\right] x^{k}$ and $d^{k}$ as

$$
d_{j}^{k}= \begin{cases}0 & \text { if } j \in J, x_{j}^{k}=0 \text { and } \nabla \lambda\left(x^{k}\right)_{j}<0, \\ \nabla \lambda\left(x^{k}\right)_{j} & \text { otherwise. }\end{cases}
$$

(2) Let

$$
\begin{aligned}
& \tau_{k}=\min \left\{\tau \in \mathbb{N} \mid\left(x^{k}+\frac{1}{2^{\tau}} d^{k}\right)_{J} \geq 0 \text { and } \lambda\left(x^{k}+\frac{1}{2^{\tau}} d^{k}\right)-\lambda\left(x^{k}\right) \geq \frac{\varepsilon}{2^{\tau}}\left\|d^{k}\right\|^{2}\right\}, \\
& \mu_{k}=\frac{1}{2^{\tau_{k}}}, \\
& y^{k+1}=x^{k}+\mu_{k} d^{k}, \\
& x^{k+1}=\frac{y^{k+1}}{\left\|y^{k+1}\right\|} .
\end{aligned}
$$

(3) Set $k \longleftarrow k+1$ and go back to step (1).

Proposition 15. The Projected Steepest Ascent Method converges to a solution of the symmetric (GEiCP).

Proof. First notice that the direction $d^{k}$ computed in step (2) is either an ascent direction or zero. This happens since $\nabla \lambda\left(x^{k}\right)^{T} d^{k}=\left(d^{k}\right)^{T} d^{k}>0 \Longleftrightarrow d^{k} \neq 0$.

The renormalization in step (3) can be done, since $y^{k+1} \neq 0$. This follows from the fact that $\left(x^{k}\right)^{T} d^{k}=\left(x^{k}\right)^{T} \nabla \lambda\left(x^{k}\right)=\frac{2}{\left(x^{k}\right)^{T} B x^{k}}\left[\left(x^{k}\right)^{T} A x^{k}-\lambda\left(x^{k}\right)\left(x^{k}\right)^{T} B x^{k}\right]=0$. Furthermore, the renormalization preserves the value of the objective function,

$$
\lambda\left(x^{k+1}\right)=\lambda\left(\frac{y^{k+1}}{\left\|y^{k+1}\right\|}\right)=\lambda\left(y^{k+1}\right),
$$

as well as the constraint $x_{J}^{k} \geq 0$. Therefore the sequence $\left\{x^{k}\right\}$ is well defined and belongs to the compact set $\left\{x \mid x_{J} \geq 0,\|x\|=1\right\}$.

For any convergent subsequence $x^{k} \longrightarrow x^{*}$, it is clear that $\nabla \lambda\left(x^{k}\right) \longrightarrow \nabla \lambda\left(x^{*}\right)$ and $d^{k} \longrightarrow d^{*}$ where

$$
d_{j}^{*}= \begin{cases}0 & \text { if } j \in J, x_{j}^{*}=0 \text { and } \nabla \lambda\left(x^{*}\right)_{j}<0, \\ \nabla \lambda\left(x^{*}\right)_{j} & \text { otherwise. }\end{cases}
$$

It is proved in the sequel that $d^{*}=0$. In fact, by the Armijo step-length choice there exists a constant $C>0$ such that $\lambda\left(y^{k+1}\right)-\lambda\left(x^{k}\right) \geq C\left\|d^{k}\right\|^{2}$. This is a canonical proof [3, Theorem 8.6.3] that uses the Mean-Value Theorem, the CauchySchwarz inequality and the Lipschitz-continuity of $\nabla \lambda(\cdot)$. Since $\left\|x^{k+1}-x^{k}\right\| \longrightarrow 0$, it follows that $\theta^{k} \longrightarrow 0$, where $\theta^{k}$ is the angle between $x^{k+1}$ and $x^{k}$.

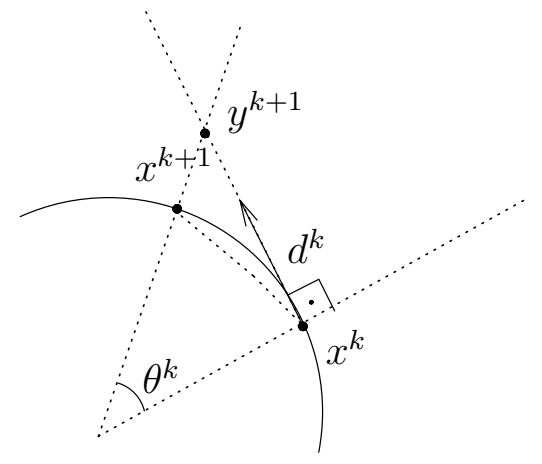

Therefore

$$
\cos \theta^{k}=\frac{\left\|y^{k+1}-x^{k}\right\|}{\left\|x^{k}\right\|}=\left\|y^{k+1}-x^{k}\right\| \longrightarrow 0 .
$$


Since $\lambda(\cdot)$ is continuous, $\lambda\left(y^{k+1}\right)-\lambda\left(x^{k}\right) \longrightarrow 0$. Since $\left\|d^{k}\right\|^{2}<\frac{1}{C}\left(\lambda\left(y^{k+1}\right)-\lambda\left(x^{k}\right)\right)$, it follows that $\left\|d^{k}\right\|^{2} \longrightarrow 0$; that is, $d^{*}=0$.

Since $\nabla \lambda\left(x^{*}\right)_{\bar{J}}=d_{\bar{J}}^{*}=0$ and $\nabla \lambda\left(x^{*}\right)_{J} \leq 0$, it follows that $x=x^{*}, \alpha=0$ and $u=-\nabla \lambda\left(x^{*}\right)$ solve the Kuhn-Tucker conditions for the (OGEiCP) ${ }_{J}$, and since $\lambda\left(x^{*}\right) \geq \lambda\left(x^{0}\right)>0$, this is a solution of the $(\mathrm{GEiCP})_{J}$.

The above algorithm has been chosen for clarity of exposition. Other line search strategies may be used, as well as other ascent directions $d^{k}$ (such that $\nabla \lambda\left(x^{k}\right)^{T} d^{k}>$ $0)$. It must be added that the line search in step (2) can be solved by exact maximization, since the derivative of the function $\hat{\lambda}(\mu)=\lambda\left(x^{k}+\mu d^{k}\right)$ is zero if and only if $\mu$ is a root of a polynomial of order 3 , as can easily be seen. Therefore at most four evaluations of the function $\hat{\lambda}(\cdot)$ are needed, namely the three roots mentioned above and the point $x^{k}+\bar{\mu} d^{k}$ corresponding to the maximal step-length $\bar{\mu}$ such that $\left(x^{k}+\bar{\mu} d^{k}\right)_{J} \geq 0$.

\section{Computational experience}

In order to compare the original formulation of the (GEiCP) as a mixed NCP and its reformulation as a nonlinear programming problem (OGEiCP), with respect to the relative difficulty of solution, two commercial packages have been chosen within the GAMS 2.5E system: GAMS/PATH 4.3 for mixed NCPs, and GAMS/MINOS 5.5 for NLPs.

The test problems were given by 80 randomly generated matrices $A \in \mathbb{R}^{n \times n}$, grouped in small-sized matrices $(n=10,20,30,40)$ and medium to large-sized matrices $(n=100,200,300,400)$. For each such value of $n$, ten matrices have been generated. Each matrix was forced to satisfy $A_{11}>0$, which gives a convenient starting solution $x^{0}=(1,0, \ldots, 0)^{T} \geq 0$ such that $\lambda\left(x^{0}\right)>0$. The corresponding problems, taking $B=I$ and $J=\{1,2, \ldots, n\}$, were given as input for both software packages. The tests were run in a Pentium II $333 \mathrm{MHz}$, running MS-Windows 98.

The PATH algorithm is an implementation of a Nonsmooth Damped Newton Method [6]. This method is applied to a reformulation of the mixed NCP as a generalized equation. The MINOS algorithm is loosely related to the Projected Steepest Ascent Method presented above and combines a Reduced-Gradient Method with a Quasi-Newton Method [10].

The computational experience is summarized by the following table. The measures for the number of iterations and running time are average measures over the ten problems in the corresponding dimension. For the PATH algorithm, major iterations correspond to Newton steps, and minor iterations are pivot steps in Lemke's Method used to construct a piecewise-linear path from an iterate to the corresponding Newton point. Columns 4, 5 and 6 are the number of problems solved without restarts, the number of problems solved using up to three restarts from distinct initial points, and the number of problems not solved by the PATH algorithm, respectively. Note that the convergence of the PATH method is only established under the hypothesis of local inversibility of the jacobians defining the $\mathrm{NCP}$, which does not hold for the (GEiCP).

Note that the PATH algorithm, without restarts, fails in $30 \%$ to $70 \%$ of the small-sized tests. Thanks to the restart mechanism and the heuristics employed, a considerable portion of these problems has been eventually solved. None of the medium and large-sized tests have been solved by the PATH algorithm. The MINOS 


\begin{tabular}{|c|c|c|c|c|c|c|c|c|}
\hline \multirow[b]{2}{*}{ 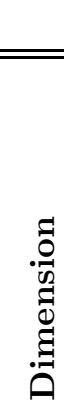 } & \multicolumn{6}{|c|}{ РАTH } & \multicolumn{2}{|c|}{ MINOS } \\
\hline & 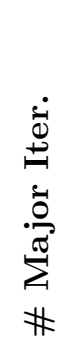 & 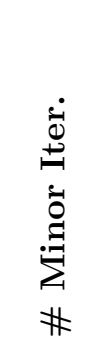 & $\begin{array}{l}\text { D } \\
\text { D } \\
\text { D } \\
0 \\
0 \\
0 \\
\text { wn } \\
\text { \# }\end{array}$ & 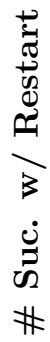 & 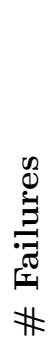 & 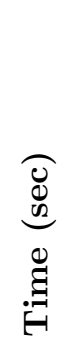 & $\begin{array}{l}\dot{\vec{\Phi}} \\
\stackrel{ \pm}{ \pm} \\
\#\end{array}$ & 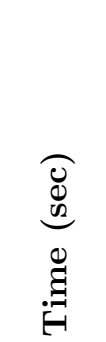 \\
\hline 10 & 33.6 & 165.5 & 7 & 2 & 1 & 0.30 & 16.8 & 0.04 \\
\hline 20 & 47.9 & 588.7 & 7 & 3 & - & 0.39 & 35.6 & 0.05 \\
\hline 30 & 77.1 & 958.8 & 4 & 6 & - & 0.71 & 52.5 & 0.09 \\
\hline 40 & 93.9 & 2418.7 & 3 & 6 & 1 & 1.30 & 67.8 & 0.15 \\
\hline 100 & - & - & - & - & $\overline{10}$ & - & 162.4 & 3.63 \\
\hline 200 & - & - & - & - & 10 & - & 324.0 & 23.96 \\
\hline 300 & - & - & - & - & 10 & - & 451.8 & 62.61 \\
\hline 400 & - & - & - & - & 10 & - & 619.9 & 120.43 \\
\hline
\end{tabular}

algorithm, on the other hand, has always found a solution, with running times ranging from 7.5 to 8.7 times faster for the small tests. The number of iterations for the MINOS algorithm is roughly linear as a function of $n$ (ranging from $1.5 n$ to $1.8 n)$.

It is possible to conclude from the above experience that the MINOS algorithm applied to the (OGEiCP) performs better than the PATH algorithm applied to the original formulation of the (GEiCP), with respect to running time and robustness (in finding a solution). It is important to add that the PATH algorithm is considered to be the most robust procedure for solving mixed complementarity problems. So the numerical results of this experience clearly indicate the relevance of the optimization formulation for finding a solution of the (GEiCP) in the symmetric case.

\section{The ASYMMETRIC CASE FOR $n=2$}

In this section the (EiCP) with $B=I$ is considered, without the symmetry assumption on the matrix $A$. It is shown that the existence of a solution when all the diagonal elements of $A$ are positive and the number of $\lambda$-solutions established for symmetric matrices also hold for asymmetric matrices of order 2 .

Proposition 16. If $A$ is a $2 \times 2$-matrix, then the (EiCP) has at most three $\lambda$ solutions.

Proof. The upper bound given by Proposition 3 is 4 . If $A_{i i} \leq 0$ for some $i$, or if a column of $A$ is positive, then the solutions corresponding to $I=\{i\}$ or $I=\{j\}$ are infeasible, and so the result follows.

On the other hand, if $A_{i i}>0$ and $A_{i j} \leq 0$, for all $i \neq j$, then the solutions corresponding to $I=\{i\}$ or $I=\{j\}$ are feasible. The remaining case is $I=\{1,2\}$, for which it is shown next that at most one solution is feasible. 
The characteristic polynomial of $A$

$$
f(\lambda)=\operatorname{det}\left(\begin{array}{cc}
\lambda-A_{11} & -A_{12} \\
-A_{21} & \lambda-A_{22}
\end{array}\right)=\lambda^{2}-\left(A_{11}+A_{22}\right) \lambda+\left[A_{11} A_{22}-A_{12} A_{21}\right]
$$

has at most two positive roots. Furthermore,

$$
f\left(A_{11}\right)=f\left(A_{22}\right)=-A_{12} A_{21} \leq 0 .
$$

If $A_{12} A_{21}=0$, then either $A_{11}$ or $A_{22}$ is an eigenvalue of $A$, with the same solution of the cases $I=\{i\}$ or $I=\{j\}$. Therefore the (EiCP) has at most three $\lambda$-solutions.

Now suppose that $A_{12}, A_{21}<0$. Then $f\left(A_{11}\right)=f\left(A_{22}\right)<0$, and there is an eigenvalue $\lambda>\max \left\{A_{11}, A_{22}\right\}>0$. The corresponding eigenvector $x$ must satisfy

$$
\begin{gathered}
\left(\lambda-A_{11}\right) x_{1}=A_{12} x_{2}, \\
x_{1}+x_{2}=1, \\
x_{1}, x_{2} \geq 0 .
\end{gathered}
$$

This is impossible, since $A_{12}<0$ and $\lambda>A_{11}$. Therefore this $\lambda$ is not a solution of the (EiCP), and the maximal number of $\lambda$-solutions is three.

Proposition 17. If $A$ is a matrix of order 2 with positive diagonal elements, then the $(\mathrm{EiCP})$ is solvable.

Proof. If there is some $j \neq i$ such that $A_{i j} \leq 0$, then the result follows. Otherwise, $A_{i j}>0, \forall i, j$, and $f\left(A_{11}\right)=f\left(A_{22}\right)=-A_{12} A_{21}<0$, where, as before, $f(\lambda)$ is the characteristic polynomial of $A$. Then there is some eigenvalue $\lambda>\max \left\{A_{11}, A_{22}\right\}>$ 0 . Since $A_{12}>0$, the following system has a solution $\left(x_{1}, x_{2}\right)$,

$$
\begin{gathered}
\left(\lambda-A_{11}\right) x_{1}=A_{12} x_{2}, \\
x_{1}+x_{2}=1, \\
x_{1}, x_{2} \geq 0 .
\end{gathered}
$$

This solution satisfies $A_{21} x_{1}=\left(\lambda-A_{22}\right) x_{2}$, since $\lambda$ is an eigenvalue of $A$. Therefore, the $(\mathrm{EiCP})$ is solvable.

This result implies that the (EiCP) is solvable when $A$ is a strictly copositive or a P-matrix (in particular, when $A$ is positive definite) of order 2. Notice that all these classes of matrices satisfy the necessary condition for the solvability of the $(\mathrm{EiCP})$

$$
\exists x \geq 0 \text { such that } x^{T} A x>0
$$

stated before. Furthermore they are sufficient for the symmetric case. More research is required to investigate whether these classes of matrices are sufficient for the solvability of the (EiCP) in the case of a general asymmetric matrix.

\section{Conclusions And Future WORK}

In this paper the Eigenvalue Complementarity Problems introduced in [5] have been considered. It has been shown that the symmetric cases are solvable with optimization techniques applied to the Rayleigh quotient subject to linear constraints. The initialization for the optimization process is a NP-complete problem, but for the majority of practical cases the initial point can be found by inspection.

Some preliminary work on the general case with asymmetric matrices of order 2 concerning the characterization of solvability and the number of $\lambda$-solutions has 
been presented. The generalization of these results for asymmetric matrices of any order is the subject of ongoing research.

\section{REFERENCES}

1. G. Auchmuty, Unconstrained variational principles for eigenvalues of real symmetric matrices, SIAM J. Math. Anal., 20(5):1186-1207, 1989. MR 91b:49055

2. G. Auchmuty, Globally and rapidly convergent algorithms for symmetric eigenproblems, SIAM J. Matrix Anal. Appl., 12(4):690-706, 1991. MR 92h:65062

3. M. S. Bazaraa, H. D. Sherali and C. M. Shetty, Nonlinear programming: theory and algorithms, 2nd Edition. John Wiley and Sons, New York, 1993. MR 80f:90110

4. F. Chatelin, Eigenvalues of matrices. John Wiley \& Sons, 1993. MR 94d:65002

5. A. P. Costa, I. N. Figueiredo, J. Júdice and J. A. C. Martins, A complementarity eigenproblem in the stability analysis of finite dimensional elastic systems with frictional contact, In.: Complementarity: applications, algorithms and extensions, edited by M. Ferris, J. S. Pang and O. Mangasarian, Kluwer, New York, pp. 67-83, 2001. MR 2002a:74094

6. S. Dirkse and M. Ferris, The PATH solver: a nonmonotone stabilization scheme for mixed complementarity problems, Optimization and Software, 5:123-156, 1995.

7. P. T. Harker and J.-S. Pang, Finite-dimensional variational inequality and nonlinear complementarity problems: a survey of theory, algorithms and applications, Math. Program. - Series $B$ - Variational inequality problems, 48(2):161-220, 1990. MR 91g:90166

8. J. Júdice, Algorithms for Linear Complementarity Problems, In.: Algorithms for Continuous Optimization, edited by E. Spedicato, Kluwer, Amsterdam, pp. 435-474, 1994. MR 95m:90133

9. M. Mongeau and M. Torki, Computing eigenelements of real symmetric matrices via optimization, Technical Report MIP 99-54. Université Paul Sabatier, Toulouse, 1999.

10. B. A. Murtagh and M. A. Saunders, MINOS 5.1 user's guide, Report SOL 83-20R, Department of Operations Research, Stanford University, 1987.

11. K. G. Murty, Linear complementarity, linear and nonlinear programming, Heldermann Verlag, Berlin, 1988. MR 89h:90240

12. J. M. Ortega, Matrix analysis: a second course, Plenum Press, New York, 1987. MR 88a:15002

13. B. N. Parlett, The symmetric eigenvalue problem, Classics in Applied Mathematics 20, SIAM, 1997. MR 99c:65072

14. A. Seeger, Eigenvalue analysis of equilibrium processes defined by linear complementarity conditions, Linear Algebra and its Applications, 292:1-14, 1999. MR 2000c:90098

Computer Science Department, University of São Paulo, Rua do Matão 1010, 05508090 São Paulo, SP, Brazil

E-mail address: mqz@ime.usp.br

Mathematics Department, University of Coimbra, 3000 Coimbra, Portugal

E-mail address: Joaquim. Judice@co.it.pt

Computer Science Department, University of São Paulo, Rua do Matão 1010, 05508090 São Paulo, SP, Brazil

E-mail address: chumes@usp.br 